EPTCO-99-001

\title{
Single-Spin Asymmetries for Small-Angle Pion Production in High-Energy Hadron Collisions
}

(submitted to Eur. Phys. J C)

\author{
Azad Ahmedov ${ }^{1, *}$, Igor V. Akushevich ${ }^{2, \dagger}$, \\ Eduard A. Kuraev ${ }^{1, \ddagger}$ and Philip G. Ratcliffe ${ }^{3, \S}$ \\ 1 Joint Inst. for Nuclear Research, 141980, Dubna, Moscow region, Russia \\ 2 Nat. Center of Particle and High Energy Physics, Bogdanovich Str. 153, \\ 220040 Minsk, Belarus \\ 3 Dip. di Scienze CC.FF.MM., Univ. degli Studi dell'Insubria, via Lucini 3, \\ 22100 Como, Italy and Ist. Naz. di Fisica Nucleare-sezione di Milano
}

February 1999

\begin{abstract}
Within the framework of a simple model, we study single-spin asymmetries for pion production in hadron-hadron collisions at highenergies with one hadron polarised. The asymmetries are generated via a mechanism of final (initial) state interactions. For peripheral kinematics, when the pion belongs to the fragmentation region of the polarised proton, we find non-zero asymmetries in the high-energy limit. Numerical results and comparision with existing experimental data are presented. We also discuss the relationship with odderon exchange phenomenology.
\end{abstract}

\footnotetext{
${ }^{*}$ E-mail: ahmedov@sunse.jinr.ru

${ }^{\dagger}$ E-mail: aku@hep. by

${ }^{\ddagger}$ E-mail: kuraev@thsun1.jinr.ru

$\S$ E-mail: pgr@fis.unico.it
} 


\section{Introduction}

Single-spin correlations have been the subject of theoretical [1-15] and experimental 16 20] study since the seventies. Earlier theoretical work paid attention mainly to time-reversal invariance violation in hadron-scattering processes. No such effect was found whereas C-odd single-spin correlation asymmetries had been observed at the level of $\sim 10 \%$ in SLAC experiments with 10-12 GeV electrons and positrons scattering off polarised protons, with, however, large error bars and later in Fermilab experiments at higher energies. The capabilities of modern CERN and DESY experiments permit the reduction of these errors due to much improved statistics. We argue here that, at small momentum transfer, large effects may be understood in the framework of pomeron and odderon exchange models and may thus provide an independent method of studying the characteristics of such exchanges in high-energy pheripheral hadron scattering.

The appearance of single-spin correlations and associated asymmetries in differential cross-sections is due to a quantum effect of interference between real and imaginary parts of different amplitudes. In phenomenological approaches, the amplitudes have been used in a Breit-Wigner form and the asymmetries turn out to be proportional to the width-to-mass ratio of the

resonance [4, 5]. For the case of polarised proton-proton collisions, with the production of pions through some intermediate nucleon resonance state in peripheral kinematics (PK), the asymmetry may be as large as 20-40\% [6]. Another mechanism for the generation of imaginary parts in scattering amplitudes is due to initial- or final-state interactions. In lowest-order perturbation theory such contributions can arise from the interference between the Born amplitude and one-loop amplitudes with a non-zero s-channel imaginary part [四].

A similar phenomena has been found for the case of large-angle production: for the kinematics of large $p_{T}$ and large $x_{F}$ of the detected hadron, single-spin asymmetries are generated by twist-3 parton correlation functions constructed from quark and gluon fields. For this case, the theoretical approach within the framework of perturbative QCD has been recently discussed by several authors [2, 3, 17, 14, 15].

Here we consider peripheral kinematics, when the unpolarised proton produces a jet moving along the initial direction of motion, which is then not detected, whereas the jet produced by the polarised proton contains a detected pion. The asymmetry originates from a term $i \epsilon^{p_{1} p_{2} l a}=\frac{i}{2} s\left[\mathbf{l}_{\wedge} \mathbf{a}\right]_{z}$, where $p_{1}$ and $p_{2}$ are the 4 -momenta of the initial protons, $l$ is the momentum of the pion in the center-of mass system (CMS) and $a$ is the spin 4-vector of the proton with momentum $p_{2}$, which is essentially a 2 -component vector 
located in the plane transverse to the beam axis (z-direction of the initialstate proton with momentum $p_{1}$ ), and $s=4 E^{2}$ is the square of the total CMS energy.

For high enough energies, the description in terms of Regge trajectories is more convenient since, for small enough momentum transfer, the contribution of operators of all twists will be of the same order of magnitude. Here the even (spin-independent) part of the differential cross-section is determined by Pomeron exchange, whereas the spin-dependent part, arising from interference between one- and two-gluon exchange amplitudes, should be described by the odderon trajectory. Note that quark exchange in the $t$-channel only gives a small contribution, suppressed by a factor of $\mathrm{m}^{2} / \mathrm{s}$, where $m$ is the proton mass. Thus, the study of single-spin asymmetries in PK may provide information on the odderon intercept. In this paper, using a QED-like framework, with the point-like hadrons, we calculate the asymmetry, defined as follows:

$$
\begin{aligned}
A & =\frac{d \sigma(a, l)-d \sigma(-a, l)}{d \sigma(a, l)+d \sigma(-a, l)} \\
& =\alpha_{\mathrm{QED}} \frac{\left(\mathbf{a}_{\wedge} \mathbf{l}\right)_{z}}{m} \mathcal{A}(r, x),
\end{aligned}
$$

where $\alpha_{\mathrm{QED}}=1 / 137$ is the $\mathrm{QED}$ coupling constant and the resolving power, $\mathcal{A}(r, x)$, is a function of $r=l_{\perp} / m$ (the transverse momentum of pion in units of the proton mass, $m$ ) and $x=2 l_{0} / \sqrt{s}$ (its energy fraction, $x \sim \mathrm{O}(1)$ ). We shall show that $\mathcal{A}(r, x)$ is a smooth rising function of $x$ of order unity. Thus, naively replacing the QED coupling constant by that of QCD or by the pomeron or odderon coupling with the proton, we find that the asymmetry may be large for large enough values of $l_{\perp}$, in qualitative agreement with experimental data [16 18,20].

The paper is organised as follows. In Section 2 we calculate the expressions for the squared matrix elements summed over spin states for processes of neutral- and charged-pion production in the framework of our QED-like approach. The corresponding charge-odd interferences for these channels are considered in Section 3, where first we obtain the expressions for asymmetries in an exclusive set-up (when both the nucleon and pion from the jet developing along the direction of polarised proton are fixed in the experiment) and then the ratio of odd and even parts of the cross-section averaged over final proton momenta are estimated. In conclusion, we discuss the situation when a hadron is detected in the opposite direction and also discuss the role of higher-order perturbation theory contributions and the relationship to odderon exchange. 


\section{Calculation of the Cross-Section}

Consider first the process

$$
P\left(p_{1}\right)+P\left(p_{2}\right) \rightarrow P\left(p_{1}^{\prime}\right)+P\left(p_{2}^{\prime}\right)+\pi_{0}(l),
$$

for which the relevant Born-approximation diagrams are show in fig. 11. We
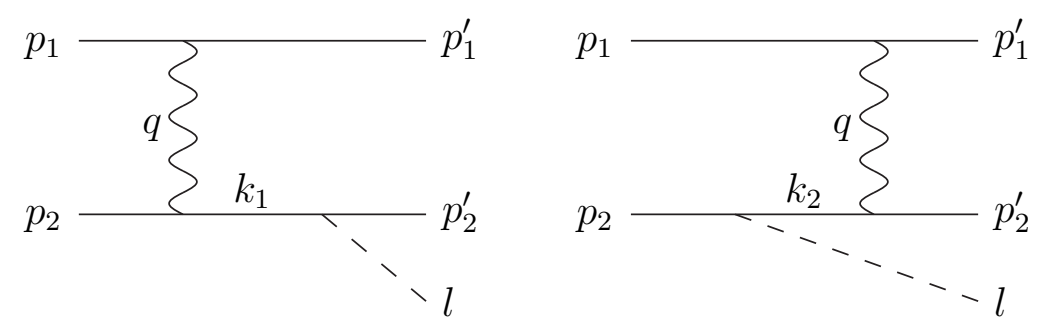

Figure 1: The amplitudes for the process $p p \rightarrow p p \pi$ (a) in the Born approximation; the proton with momentum $p_{2}$ is polarised.

shall use the Sudakov expansion for the momenta of the problem, introducing the almost light-like vectors

$$
\begin{aligned}
& \tilde{p}_{1}^{\mu}=p_{1}^{\mu}-\frac{m^{2}}{s} p_{2}^{\mu}, \\
& \tilde{p}_{2}^{\mu}=p_{2}^{\mu}-\frac{m^{2}}{s} p_{1}^{\mu} .
\end{aligned}
$$

With these we define the following parametrisation of the momenta in the problem:

$$
\begin{aligned}
q^{\mu} & =p_{1}^{\mu}-p_{1}^{\prime \mu}=\alpha \tilde{p}_{2}^{\mu}+\beta \tilde{p}_{1}^{\mu}+q_{\perp}^{\mu}, \\
p_{2}^{\prime \mu} & =(1-x) \tilde{p}_{2}^{\mu}+\beta^{\prime} \tilde{p}_{1}^{\mu}+p_{\perp}^{\mu}, \\
l^{\mu} & =x \tilde{p}_{2}^{\mu}+\beta_{l} \tilde{p}_{1}^{\mu}+l_{\perp}^{\mu} \\
q_{\perp}^{\mu} & =p_{\perp}^{\mu}+l_{\perp}^{\mu}
\end{aligned}
$$

The transverse parts, $v_{\perp}$, obey

$$
\begin{gathered}
v_{\perp} \cdot p_{1}=0=v_{\perp} \cdot p_{2} \\
v_{\perp}^{2}=-\mathbf{v}^{2}<0 .
\end{gathered}
$$

We have also used the specific properties of PK: the sum of energy fractions of pion and proton from the jet moving along initial polarised proton is equal 
to unity, $x \sim \mathrm{O}(1)$; moreover, the reality conditions for final particles permits the neglect of the "small" components of momenta:

$$
\begin{aligned}
& s \beta^{\prime}=\frac{m^{2}+\mathbf{p}_{2}^{2}}{1-x}, \\
& s \beta_{l}=\frac{\mathbf{l}^{2}}{x} .
\end{aligned}
$$

Here and in what follows we neglect the pion mass squared compared to that of the proton. The intermediate fermion denominators in the Born graphs (see Fig. 1) are then given by

$$
\begin{aligned}
& d_{1}=k_{1}^{2}-m^{2} \simeq \frac{m^{2} x^{2}+(x \mathbf{q}-\mathbf{l})^{2}}{x(1-x)}, \\
& d_{2}=k_{2}^{2}-m^{2} \simeq-\frac{m^{2} x^{2}+\mathbf{l}^{2}}{x} .
\end{aligned}
$$

Note that $k_{1}^{2}$ is just the invariant-mass squared of the jet moving along $\mathbf{p}_{2}$. We shall show that the dominant contribution arises when this quantity is of the order of some nucleon mass squared.

In the Born approximation, the matrix element has the following form:

$$
\mathcal{M}_{\pi^{0}}=\frac{4 \pi \alpha g}{q^{2}} J_{\mu}^{(1)}\left(p_{1}\right) D^{\mu \nu}(q) J_{\nu}^{(2)}\left(p_{2}\right)
$$

where $\alpha=\alpha_{\mathrm{QED}}, g$ is the pion-nucleon coupling constant and $D^{\mu \nu}$ is the exchange photon polarisation tensor. The current vectors introduced are

$$
\begin{aligned}
J_{\mu}^{(1)}\left(p_{1}\right) & =\bar{u}\left(p_{1}^{\prime}\right) \gamma_{\mu} u\left(p_{1}\right), \\
J_{\nu}^{(2)}\left(p_{2}\right) & =\bar{u}\left(p_{2}^{\prime}\right) O_{\nu} u\left(p_{2}\right)
\end{aligned}
$$

where

$$
\begin{aligned}
O_{\nu} & =\frac{1}{d_{1}} \gamma_{5}\left(\not p_{2}^{\prime}+\not l+m\right) \gamma_{\nu}+\frac{1}{d_{2}} \gamma_{\nu}\left(\not \not_{2}-\not \partial+m\right) \gamma_{5} \\
& =\gamma_{5}\left[\frac{\not \gamma_{\nu}}{d_{1}}-\frac{\gamma_{\nu} \not \ell}{d_{2}}\right]
\end{aligned}
$$

In the last step of (13) we have used the Dirac equation for free protons. For PK, only the so-called "nonsense" components of the decomposition of photon polarisation tensor give a non-vanishing contribution in the highenergy limit:

$$
\begin{aligned}
D^{\mu \nu} & =g_{\perp}^{\mu \nu}+2\left(p_{1}^{\mu} p_{2}^{\nu}+p_{1}^{\nu} p_{2}^{\mu}\right) / s \\
& \simeq 2 p_{1}^{\nu} p_{2}^{\mu} / s .
\end{aligned}
$$


Further simplifications may be made using the current conservation condition, $q \cdot J^{(2)}=0$, which implies $p_{1} \cdot J^{(2)} \simeq-q_{\perp} \cdot J^{(2)} / \beta$.

As a result, the matrix element in the Born approximation becomes

$$
\mathcal{M}_{\pi^{0}}=-\frac{8 \pi \alpha g}{\beta s q^{2}} \bar{u}\left(p_{1}^{\prime}\right) \not p_{2} u\left(p_{1}\right) \bar{u}\left(p_{2}^{\prime}\right) O u\left(p_{2}\right),
$$

where $O=q_{\perp}^{\nu} O_{\nu}$. Here the exchange denominator is $q^{2}=-\left(\mathbf{q}^{2}+q_{m}^{2}\right)$, with $q_{m}^{2}=m^{2}(\tilde{s} / s)^{2}$ and $\tilde{s}=\left(m^{2} x+\mathbf{p}_{2}^{2}\right) /(1-x)+\mathbf{l}^{2} / x$. However, in order to regulate the infrared divergences, we shall use a massive vector-particle propagator: $q^{2}=-\left(\mathbf{q}^{2}+\mu^{2}\right)$. For the modulus squared of the Born matrix element, summed over spin states, we thus obtain

$$
\sum_{\text {spins }}\left|\mathcal{M}_{\pi_{0}}\right|^{2}=(16 \pi g \alpha)^{2} \frac{s^{2} x^{2} \mathbf{q}^{2}}{d_{1}\left(-d_{2}\right)\left(\mathbf{q}^{2}+\mu^{2}\right)^{2}} .
$$

Note that here we consider the hadrons as point-like particles and so the high-frequency contributions are not negligible (unrealistically so). Thus, although all integrals are in fact convergent, we introduce a form-factor of the form $\exp \left(-b \mathbf{q}^{2}\right)$, with $b \sim 1 \mathrm{GeV}^{-2}$. As is well known, single-spin correlation effects are absent in the Born approximation, as a consequence of the reality of Born amplitudes and the form of the proton spin-density matrix:

$$
\begin{aligned}
u\left(p_{2}, a\right) \bar{u}\left(p_{2}, a\right) & =\left(\not p_{2}+m\right)\left(1+\gamma_{5} \not \phi\right), \\
\operatorname{tr}\left[\gamma_{5} \not \not \not b \not \subset \not d\right] & =4 i \epsilon^{a b c d} .
\end{aligned}
$$

It is also well known that in the case of elastic small-angle chargedparticle scattering, the Born amplitude acquires a Coulomb phase factor, $\exp \left[i \alpha \pi \ln \left(-q^{2} / \mu^{2}\right)\right]$, when multiphoton exchange is taken into account. A similar factor appears in the case of inelastic processes in PK, such as those we are considering here.

\section{Calculation of the Spin Dependence}

For the spin-dependent part of the interference between single- and doublephoton exchange amplitudes (see Fig. 2), a calculation similar to that performed above gives

$$
\begin{aligned}
\sum_{\text {spins }} \Delta\left|\mathcal{M}_{\pi_{0}}\right|^{2} & =\frac{2^{11} \pi^{2} \alpha^{3} g^{2} s}{\tilde{s}^{2}|\mathbf{q}|^{4}} \ln \left(\frac{|\mathbf{q}|^{2}}{\mu^{2}}\right) \\
& \times \frac{i}{4} \operatorname{tr}\left[-\gamma_{5} \not \alpha \tilde{O}\left(\not p_{2}^{\prime}+m\right) O\left(\not p_{2}^{\prime}+m\right) \not p_{1}\left(\not p_{2}+m\right)\right],
\end{aligned}
$$




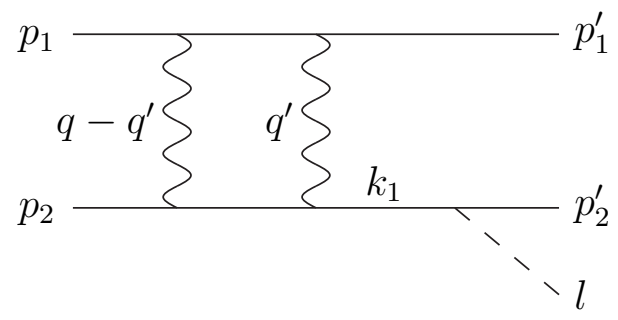

Figure 2: An example higher-order contribution to the process $p p \rightarrow p p \pi$, as considered in the text.

where again $O=q_{\perp}^{\nu} O_{\nu}$. After calculating the trace we obtain, for the exclusive set-up,

$$
\begin{aligned}
A & =\frac{\Delta \sum\left|\mathcal{M}_{\pi_{0}}\right|^{2}}{\sum\left|\mathcal{M}_{\pi_{0}}\right|^{2}} \\
& =4 \alpha \ln \left(\mathbf{q}^{2} / \mu^{2}\right) m|\mathbf{a}||\mathbf{q}| \sin \phi_{q} \frac{[x \tilde{s}-2 \mathbf{q} \cdot \mathbf{l}]\left[x \mathbf{q}^{2}+(1-x) 2 \mathbf{q} \cdot \mathbf{l}\right]}{\tilde{s} d_{2} \mathbf{q}^{2}}
\end{aligned}
$$

where $\phi_{q}$ is the azimuthal angle between the transverse 2-vectors a and $\mathbf{q}$. We note that the asymmetry is finite in the small-q limit.

The differential cross-section in the Born approximation is

$$
\frac{d \sigma_{B}}{d x d r}=\frac{2 \alpha_{Q E D} \alpha_{p p \pi}}{m^{2}} \frac{r}{x(1+\rho)^{2}}\left[\ln \frac{(1+\rho)^{2}}{\sigma}-1\right],
$$

where $\alpha_{p p \pi}=g^{2} /(4 \pi) \approx 3, \rho=r^{2} / x^{2}$ and $\sigma=\left(\mu^{2}+q_{m}^{2}\right) / M^{2}$. The Born cross-section is a monotonically rising function of $x\left(\sim x^{3}\right)$ for small $x$. It falls rapidly as $1 / l^{3}$ for large pion transverse momentum $l$ and reaches the maximum value for $l_{\perp} \sim m x / \sqrt{3}$. The asymmetry in the inclusive set-up, defined as a ratio of even and odd parts of the cross-section averaged over transverse momenta, may be written, for small $\sigma$, in form:

$$
A=\frac{\int d^{2} \mathbf{q} \sum \Delta|M|^{2}}{\int d^{2} \mathbf{q} \sum|M|^{2}}=|\mathbf{a}| \sin \phi_{l} \frac{4 \alpha_{\mathrm{QED}} R}{r\left[\ln \frac{(1+\rho)^{2}}{\sigma}-1\right]},
$$

with

$$
\begin{aligned}
R= & x \ln (1+\rho) \ln \frac{1+\rho}{\sigma} \\
& +x(1-x)\left[\frac{\rho}{2(1+\rho)} \ln ^{2} \sigma+f_{1}(\rho, x) \ln \sigma+f_{2}(\rho, x)\right],
\end{aligned}
$$

where $f_{1,2}$ are rather flat functions; the complete expression for $R$ is given in the Appendix. The results of a numerical calculation of the asymmetry in 
the inclusive set-up as a function of $\mathbf{l}$ for a various of values of the Feynman variable, $x$, and as a function of $x$ for various values of $\mathbf{l}$ are presented in Fig. 3 . While the detailed dependence on $x$ and $l_{\perp}$ is not entirely reproduced, the general trends are seen to be correct.

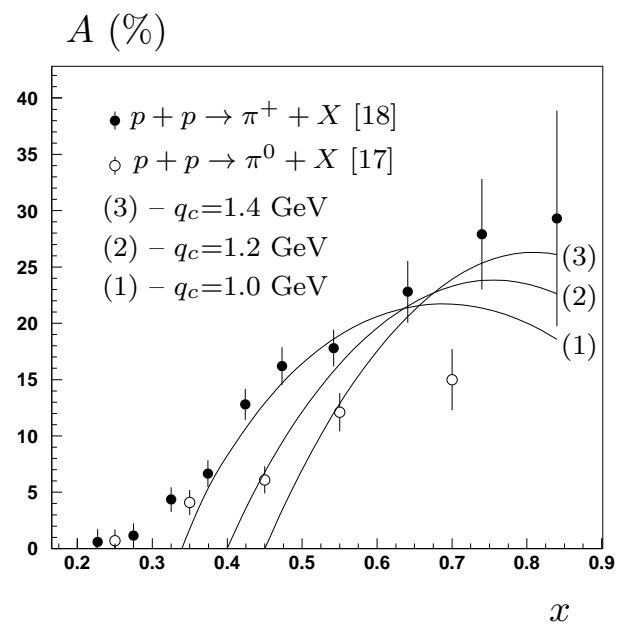

(a)

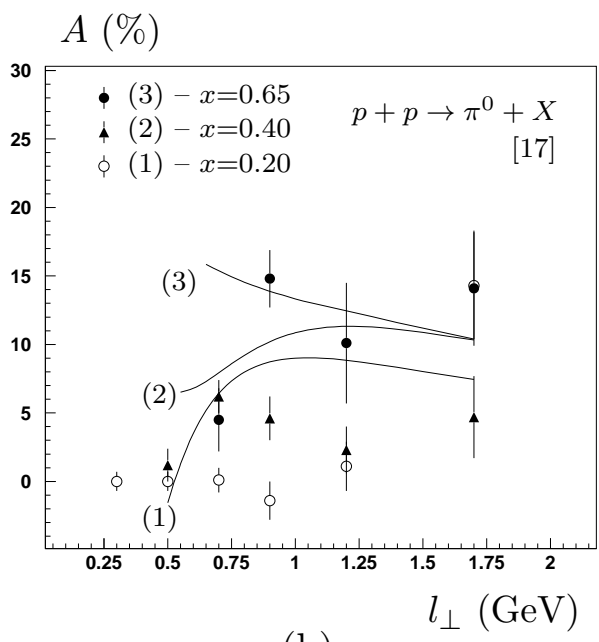

(b)

Figure 3: The model calculations for the asymmetry plotted as a function of (a) $l_{\perp}$ and (b) $x$.

A similar calculation for the case of $\pi^{+}$production in the small-q limit yields

$$
\begin{aligned}
& x^{2} \sum\left|\mathcal{M}_{\pi_{+}}\right|^{2}=(1-x)^{2} \sum\left|\mathcal{M}_{\pi_{0}}\right|^{2}, \\
& x^{2} \sum \Delta\left|\mathcal{M}_{\pi_{+}}\right|^{2}=(1-x)^{2} \sum \Delta\left|\mathcal{M}_{\pi_{0}}\right|^{2},
\end{aligned}
$$

where $\sum \Delta|\mathcal{M}|^{2}$ stands for the spin-weighted sum. Thus, the asymmetries for $\pi^{+}$production roughly coincide with those for the $\pi^{0}$ case.

\section{Conclusions}

We see that asymmetry effects due to single transverse polarisation are not suppressed in the limit of large total CMS energy, $\sqrt{s}$, in the case when the produced hadron belongs to the jet of the polarised proton. The overall normalisation depends on the detailed mechanism of vector meson (photon and gluon) interaction with nucleons and is bound to the choice of the parameters 
$\alpha$ and $\mu$. The naive replacements $\alpha \rightarrow \alpha_{s}$ and $\mu \rightarrow \Lambda_{\mathrm{QCD}}$ lead to asymmetries of the same order as those found experimentally and which grow with transverse momentum for small values. This behaviour agrees qualitatively with the existing data [16 18, 20].

It should be noted that in the case when the pion is detected in the direction of the jet moving in the opposite direction (i.e., along $\mathbf{p}_{1}$ ) the asymmetry effect will be suppressed in the $s \rightarrow \infty$ limit. In fact, information on the transverse polarisation of proton $p_{2}$ cannot be transmitted to jet components developing from proton $p_{1}$ unless at least one "sense" component of the virtual photon polarisation tensor is used, $g_{\perp}^{\mu \nu}$; consequently, it will be suppressed by powers of $\mathrm{m}^{2} / \mathrm{s.}^{1}$

In particular, for elastic proton-proton scattering where an unpolarised scattered proton with momentum $p_{1}^{\prime}$ is detected we obtain:

$$
A_{p\left(p_{1}^{\prime}\right)}=\alpha \frac{5 m \mathbf{q}^{2}\left(\mathbf{a}_{\wedge} \mathbf{q}\right)_{z}}{2 s^{2}}
$$

Higher-order QCD effects may be taken into account by introducing a factor $\left(s / s_{0}\right)^{a_{\mathrm{O}}}$ into the odd part of the elastic proton-proton scattering zeroangle amplitude, where $a_{\mathrm{O}}$ is the odderon intercept. Thus, the asymmetry considered here, associated with twice the imaginary part, acquires a factor $a^{2}\left(s / s_{0}\right)^{a_{\mathrm{O}}-a_{\mathrm{P}}}$, where $a_{\mathrm{P}}$ is the Pomeron intercept. For small-angle scattering of electrons off polarised protons, the odderon contribution manifests itself in higher orders of PT due to conversion of photons into gluons through the $\gamma \gamma \rightarrow g g$ and $\gamma g \rightarrow g g$ kernels, which may also be investigated at DESY.

\section{Acknowledgments}

One of us (E.A.K.) is grateful to Pierre Gauron, Basarab Nicolescu and Gregory P. Korchemsky for useful discussions, also to the Cariplo Foundation for Scientific Research, for financial support, and to the Department of Sciences, University of Insubria, Como, for hospitality, while this work was performed.

\section{Appendix}

After some simple operations, the odd part of the cross-section averaged over q may be put into the form:

$$
\int \frac{d^{2} \mathbf{q}}{\pi} \sum \Delta|M|^{2}=\frac{2^{10}(\pi g)^{2} \alpha_{\mathrm{QED}}^{3} x s^{2}}{d_{2}^{2}} \frac{|\mathbf{a}| \sin \phi_{l}}{r}
$$

\footnotetext{
${ }^{1}$ We are grateful to Lev N. Lipatov for discussions on this point.
} 


$$
\times \int \frac{d^{2} \mathbf{q}}{\pi} \frac{\ln \left(\mathbf{q}^{2} / \mu^{2}\right) \mathbf{q} \cdot \mathbf{l}}{\left(\mathbf{q}^{2}+\mu^{2}\right)^{2} d_{1} \tilde{s}}\left[x^{2} \mathbf{q}^{2} \tilde{s}-4(\mathbf{q} \cdot \mathbf{l})^{2}-2 x d_{2} \mathbf{q} \cdot \mathbf{l}\right],
$$

where we have used another equivalent form of the numerator in Eq. (19):

$$
[x \tilde{s}-2 \mathbf{q} \cdot \mathbf{l}]\left[x \mathbf{q}^{2}+(1-x) 2 \mathbf{q} \cdot \mathbf{l}\right]=x^{2} \mathbf{q}^{2} \tilde{s}-4(\mathbf{q} \cdot \mathbf{l})^{2}-2 x d_{2} \mathbf{q} \cdot \mathbf{l}
$$

We write the integrals of the three terms in square brackets in Eq. (25) as $J_{1,2,3}$ respectively. The quantity $R$ introduced in Eq. (22) may then be re-expressed in terms of the $J_{i}$ :

$$
R=\frac{\sum_{i=1}^{3} J_{i}}{x(1-x)}
$$

For the first term we have

$$
J_{1}=x(1-x) \int \frac{d^{2} \mathbf{q}}{\pi} \mathbf{q} \cdot \mathbf{l} \frac{\ln (1 / \sigma)+\ln \left(\mathbf{q}^{2} / m^{2}\right)}{\mathbf{q}^{2}\left(\mathbf{q}^{2}-2 \mathbf{q} \cdot \mathbf{l} / x+(1+\rho) m^{2}\right)},
$$

where $\rho=\mathrm{l}^{2} /\left(m^{2} x^{2}\right)$. To use the Feynman trick of combining the denominators, we use the following representation for the logarithm:

$$
\left(\frac{m^{2}}{\mathbf{q}^{2}}\right) \ln \left(\frac{\mathbf{q}^{2}}{m^{2}}\right)=-\left.\frac{d}{d g}\left(\frac{\mathbf{q}^{2}}{m^{2}}\right)^{-g}\right|_{g=1} .
$$

The denominators may be combined using the identity

$$
\frac{1}{u^{g} v^{h}}=\frac{\Gamma(g+h)}{\Gamma(g) \Gamma(h)} \int_{0}^{1} d z \frac{(1-z)^{g-1} z^{h-1}}{[(1-z) u+z v]^{g+h}} .
$$

The further standard procedure of performing the $d^{2} \mathbf{q}$ integration, subsequent differentiation with respect to $g$ and integration over $z$ yields

$$
J_{1}=x^{2}(1-x) \ln (1+\rho) \ln \frac{1+\rho}{\sigma} .
$$

In the evaluation of $J_{2}$ we may set $\sigma=0$ in the denominator. Joining the first two denominators we have

$$
\frac{1}{d_{1} \tilde{s}}=\frac{(1-x)^{2}}{x} \int_{0}^{1} d y\left[\mathbf{q}^{2}-2 \mathbf{q} \cdot \mathbf{l} \eta / x+\eta m^{2}(1+\rho)\right]^{-2},
$$

where $\eta=x+y(1-x)$. And following a procedure similar to that given above we obtain

$$
\begin{aligned}
J_{2}=-4[x(1-x) \rho]^{2} \int_{0}^{1} d y \eta \int_{0}^{1} d z & z^{2}(1-z) \\
\times & {\left[\frac{\rho \eta^{2} z^{2}}{D^{3}}(2 L-1)+\frac{3 L}{2 D^{2}}\right], }
\end{aligned}
$$


where $L=\ln \frac{D}{(1-z) \sigma}$ and $D=\eta(1+\rho) z-\eta^{2} z^{2} \rho+(1-z) \sigma$. We note that we may set $\sigma=0$ in the expression for $D$ although in evaluating $J_{3}$ we cannot omit $\sigma$ in the denominator. Nevertheless, using the identity

$$
\ln \left(\frac{\mathbf{q}^{2}}{\sigma m^{2}}\right)=\ln \left(\frac{\mathbf{q}^{2} / m^{2}+\sigma}{\sigma}\right)-\ln \left(1+\frac{\sigma m^{2}}{\mathbf{q}^{2}}\right),
$$

we may apply the procedure of differentiation to the first term. The second is important in the region $\mathbf{q}^{2} \sim \sigma m^{2}$ and may be evaluated explicitly:

$$
\begin{aligned}
-2 x^{2}(1+\rho) \int \frac{d^{2} \mathbf{q}}{\pi} & (\mathbf{q} \cdot \mathbf{l})^{2} \frac{\ln \left(1+\frac{\sigma m^{2}}{\mathbf{q}^{2}}\right)}{\left(\mathbf{q}^{2} / m^{2}+\sigma\right)^{2} d_{1} \tilde{s}} \\
& =-\frac{x^{2}(1-x)^{2} \rho}{1+\rho} \int_{0}^{\infty} \frac{d z z \ln \left(1+\frac{1}{z}\right)}{(1+z)^{2}} \\
& =-\frac{x^{2}(1-x)^{2} \rho}{1+\rho}\left(\frac{\pi^{2}}{6}-1\right) .
\end{aligned}
$$

The total answer for $J_{3}$ is then

$$
\begin{aligned}
J_{3}= & -\frac{x^{2}(1-x)^{2} \rho}{1+\rho}\left(\frac{\pi^{2}}{6}-1\right) \\
+ & 2 x^{3}(1-x)^{2} \rho(1+\rho) \\
& \times \int_{0}^{1} d y \int_{0}^{1} d z z(1-z)\left[\frac{\rho z^{2} \eta^{2}(2 L-1)}{D^{3}}+\frac{L}{2 D^{2}}\right] .
\end{aligned}
$$

\section{References}

[1] G.L. Kane, J. Pumplin and W. Repko, Phys. Rev. Lett. 41 (1978) 1689.

[2] J. Qiu and G. Sterman, Phys. Rev. Lett. 67 (1991) 2264.

[3] J. Qiu and G. Sterman, Nucl. Phys. B378 (1992) 52.

[4] R. Barni, G. Preparata and P.G. Ratcliffe, Phys. Lett. B296 (1992) 251.

[5] P.G. Ratcliffe, in the proc. of The X Int. Symp. on High Energy Spin Physics (Nagoya, Nov. 1992), eds. T. Hasegawa, N. Horikawa, A. Masaike and S. Sawada (Univ. Acad. Press, 1993), p. 635.

[6] A. Arbuzov et al., Phys. Atom. Nucl. 57 (1994) 1004.

[7] A.V. Efremov, V.M. Korotkiyan and O.V. Teryaev, Phys. Lett. B348 (1995) 577. 
[8] V.M. Korotkiian and O.V. Teryaev, Phys. Rev. D52 (1995) 4775.

[9] M. Anselmino, M. Boglione and F. Murgia, Phys. Lett. B362 (1995) 164.

[10] M. Anselmino, E. Leader and F. Murgia, Phys. Rev. D56 (1997) 6021.

[11] N. Hammon, O. Teryaev and A. Schäfer, Phys. Lett. B390 (1997) 409.

[12] M. Anselmino and F. Murgia, Phys. Lett. B442 (1998) 470.

[13] N. Hammon, B. Ehrnsperger and A. Schäfer, J. Phys. G24 (1998) 991.

[14] J. Qiu and G. Sterman, Phys. Rev. D59 (1999) 014004.

[15] P.G. Ratcliffe, Phys. Rev. D59 (1999) 014038.

[16] D.L. Adams et al. (FNAL-E581 collab.), Phys. Lett. B261 (1991) 201.

[17] D.L. Adams et al. (FNAL-E704 collab.), Phys. Lett. B264 (1991) 462.

[18] D.L. Adams et al. (FNAL-E581 collab.), Z. Phys. C56 (1992) 181.

[19] A. Bravar et al. (FNAL-E704 collab.), Phys. Rev. Lett. 75 (1995) 3073.

[20] D.L. Adams et al. (FNAL-E704 collab.), Nucl. Phys. B510 (1998) 3. 